\title{
EL IDEAL DE PENSAR CRÍTICAMENTE: PROMESAS Y REALIDADES
}

The ideal of critical thinking: promises and realities

O ideal de pensar criticamente: promessas e realidades

RECIBIDO: 7 DE SEPTIEMBRE DE 2012

Iván Alfonso Pinedo Cantillo (Colombia)

Fundación Educativa Gimnasio Los Caobos alfiva@hotmail.com

Magíster en Administración de Instituciones Educativas.

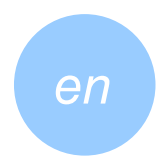

ABSTRACT

\section{RESUMEN}

El progreso humano en general y el desarrollo personal de los estudiantes están vinculados indiscutiblemente a procesos rigurosos de reflexión, pero una reflexión que en el ámbito educativo calificamos como crítica e intencionada. Educar con conciencia crítica es educar para construir humanidad mediante una orientación intelectual específica que nos hace más libres y auténticos. En este contexto, encontramos el pensamiento crítico como herramienta pedagógica privilegiada que busca fomentar la autonomía del pensamiento y la puesta en marcha de procesos inéditos de humanización, entendida como el proceso continuo que busca potencializar todas y cada una de las dimensiones del ser humano. De esta forma, reflexión intencionada, pensamiento crítico y procesos de humanización en el aula constituyen un triángulo pedagógico que puede alimentar la noble tarea de educar ciudadanos en el siglo XXI.

PALABRAS CLAVE: Reflexión, pensamiento crítico, procesos de humanización en el aula.
KEYWORDS: Reflection, critical thinking, humanization processes in the classroom.
Human progress in general and personal development of students are indisputably linked to rigorous reflection processes, but a reflection that in education we qualify as critical and intentional. Educating with critical awareness is to educate to construct humanity through a specific intellectual orientation that makes us more free and authentic. In this context, we find critical thinking as a privileged pedagogical tool that seeks to promote the autonomy of thought and the implementation of new processes of humanization, understood as the continuous process that seeks to potentiate each and every human dimension. Thus, intentional reflection, critical thinking and humanization processes in the classroom are a pedagogical triangle that can power the noble task of educating citizens in the twenty-first century.

\section{RESUMO}

O progresso humano em geral e o desenvolvimento pessoal dos estudantes estão vinculados indiscutivelmente a processos rigorosos de reflexão, mas uma reflexão que no âmbito educativo qualificamos como crítica e intencionada. Educar com consciência crítica é educar para construir humanidade mediante uma orientação intelectual específica que nos faz mais livres e autênticos. Em este contexto, encontramos o pensamento crítico como ferramenta pedagógica privilegiada que procura fomentar a autonomia do pensamento e a posta em marcha de processos inéditos de humanização, entendida como o processo contínuo que procura potencializar todas e cada uma das dimensões do ser humano. Desta forma, reflexão intencionada, pensamento crítico e processos de humanização na aula constituem um triângulo pedagógico que pode alimentar a nobre tarefa de educar cidadãos no século XXI.

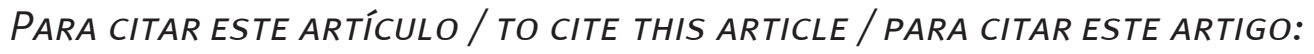



En los últimos años tenemos importantes razones para sentirnos insatisfechos con la baja calidad que manifiestan nuestros niños, adolescentes y jóvenes en el aprendizaje. Los resultados de Colombia en el 2006 y 2009 en las pruebas PISA (Programa Internacional para la Evaluación de Estudiantes), presentados por la Organización para la Cooperación y el Desarrollo Económico (OECD); la calificación obtenida en el Estudio Internacional de Tendencias en Matemáticas y Ciencias (TIMSS) de la Asociación Internacional para la Evaluación del Rendimiento Educativo (IEA); y los datos parciales obtenidos en los exámenes PIRLS (Estudio Internacional de Progreso en Comprensión Lectora) para niños de 9 y 10 años son bastante preocupantes de cara a la competitividad futura de la nación y el progreso en todos los niveles. En resumen, las pruebas indican con datos estadísticos las grandes deficiencias que el país tiene en lectura, matemáticas y ciencias. Las cifras arrojadas por estos indicadores de gestión educativa confirman en muchos aspectos los estudios que en la última década vienen desarrollando diversos investigadores universitarios, pedagogos, psicólogos y docentes que trabajan directamente en las aulas tratando de responder a la pregunta qué y cómo están aprendiendo nuestros estudiantes. Las respuestas coinciden en que un gran número de egresados de la educación secundaria y media nacional se caracterizan en su aprendizaje porque:

- No han desarrollado suficientemente la competencia cognitiva de encontrar causas, pruebas o razones que ratifiquen una idea.

- Les falta dominio de las operaciones inductivas y deductivas propias del pensamiento formal.

- Se les dificulta sustentar racionalmente sus opiniones o tienen dificultades para evaluar con juicio crítico sus propios puntos de vista o los puntos de vista de los demás.

- Presentan poca profundidad en el análisis escrito y verbal. La visión estrictamente literal del texto continúa siendo preponderante.

- Difícilmente descubren supuestos y valores ocultos en las ideas, teorías y propuestas de reflexión.

- Les falta sentido y capacidad para hacer una lectura crítica de la realidad social, política y económica que les rodea.

Estas son algunas de las conclusiones a las que han llegado diversos expertos en el tema de la educación colombiana. Como es natural, en este tipo de informes presentados por el Ministerio de Educación Nacional (MEN) y el Instituto Colombiano para el Fomento de la Educación Superior (Icfes) siempre pueden coexistir puntos de vista discutibles, igualmente, estos generan reacciones a favor y en contra de los datos provenientes de estudios escolares comparados que no tienen en cuenta las situaciones particulares de cada región, pero en términos generales son expresión de un malestar que aparece con persistencia en nuestra realidad educativa de los últimos años. ${ }^{1}$ En el contexto nacional llamaremos a esta situación de aprendizaje "carencias de habilidades de reflexión en el mundo estudiantil”. No es un tema ajeno o lejano dentro de las ideas pedagógicas actuales, puesto que cada vez hay mayor conciencia en el ámbito mundial de que la humanización del niño y el joven pasa necesariamente por el desarrollo de la capacidad reflexiva.

1 Esta información inicial se puede contrastar a la luz de los datos suministrados por la OECD: informes de resultados PISA 2003, 2006, 2009. También en los reportes técnicos internacionales de TIMSS 2003, 2007. Cf. OECD, 2004; OECD, Unesco,2003; OECD, PISA, 2010; Mullis, Martin, González y Chrostowski, 2008; Martin, Mullis y Foy, 2008. Los informes técnicos TIMSS se encuentran disponibles en: http://timss.bc.edu/timss2007/techreport.btml; http://timss.bc.edu/timss2007/frameworks.btml y http://timss.bc.edu/timss2007/intl_reports.html.

| Panorama 
De acuerdo con lo anterior, en este artículo se plantea la tesis fundamental de cómo el progreso humano en general y el desarrollo personal de los estudiantes están vinculados indiscutiblemente a procesos rigurosos de reflexión, pero de una reflexión que en el contexto que nos interesa calificamos como crítica e intencionada. Educar con conciencia crítica es educar movidos por el noble ideal de alcanzar una sociedad democrática, justa y participativa, y con la promesa de formar mejores seres humanos mediante una orientación intelectual específica que nos hace más libres y auténticos. En sentido opuesto, en la medida que nuestra capacidad reflexiva es pobre, está limitada, entonces somos seres humanos que decrecemos en nuestras potencialidades mentales y de acción.

Para comprender mejor esta orientación conviene ampliar algunas ideas. Las funciones de la reflexión pueden ser ilimitadas, pero desde el punto de vista del educador hay algunas especialmente relevantes, pues son indispensables en los procesos pedagógicos para conseguir objetivos fundamentales en el camino hacia la humanización de los estudiantes. Cuando hablamos de humanización nos referimos al "proceso continuo, permanente y participativo que busca desarrollar armónica y coherentemente todas y cada una de las dimensiones del ser humano: ética, espiritual, cognitiva, afectiva, comunicativa, estética, corporal y sociopolítica, a fin de lograr su realización plena en la sociedad" (Rincón, 2002, p. 17). Es decir, ver al ser humano como uno y a la vez como pluridimensional, bien diverso como cuerpo y a la vez plenamente integrado y articulado en una unidad.

¿Por qué plantear la humanización de esta manera? Porque en educación la referencia al sustantivo formación es un hecho constante, y si lo entendemos bien y nos alejamos de la idea de uniformidad, este nos invita a pensar en la orientación de nuestros estudiantes, es decir, en que se forma para algo, con un fin, con una intencionalidad, con un propósito. Como afirma el pedagogo José Leonardo Rincón, en educación no hay neutralidad valorativa tal como propone el paradigma científico positivista, por el contrario, se forma, se da forma, de cara a una cultura, a una sociedad, en un determinado contexto (2002). En países de América Latina a veces se piensa que la escuela educa para ser uno más dentro de la gran masa, obreros inconscientes, individuos alienados sometidos a los lineamientos del capitalismo, subyugados a las nuevas formas de ejercicio del poder y otras expresiones semejantes; en nuestra región se comparte la idea de que debemos formar no para reproducir el statu quo imperante, sino para ser hombres plenamente auténticos, que piensan por sí mismos, que son críticos, que actúan en coherencia con sus principios, que son competentes y capaces de discernir los signos de los tiempos de una forma reflexiva e investigativa (Freire, 2004).

Si esto es así, la tesis inicial resulta coherente: cuando el estudiante carece de estas herramientas de reflexión, decrece en su camino de humanización. Como bien dice Peter Facione, "sería alguien que no se preocupa seriamente por nada, que prefiere no pensar, de mente cerrada, con dificultad para entender lo que otros piensan, injusto a la hora de juzgar, en fin, alguien a quien uno no desea tener sentado al lado en un trayecto de bus largo o elegir para un cargo público" (2007, pp. 166). Ahora bien, no es que la escuela sea el único lugar de crecimiento humano, pues está la familia, el trabajo, los grupos de amigos, incluso la Iglesia; pero dadas las horas que el estudiante permanece en el colegio, el cúmulo de asignaturas a las que se enfrenta y las demás vivencias que se tienen a diario en el denominado currículo oculto, la escuela es definitivamente un lugar privilegiado de humanización.

Panorama | pp. $59-72 \mid$ Volumen 7 | Número 13 |

Como se puede entrever, en el fondo de esta propuesta se encuentra un enfoque filosófico-humanista de la educación, el cual podemos reconocer como un gran ideal y una promesa para el ser humano en los años venideros: queremos una generación distinta de seres humanos, que creciendo en habilidades de pensamiento también esté dispuesta a transformar aquellas estructuras que sumergen a miles de hombres y mujeres del continente en lo infrahumano. El común denominador de la perspectiva humanista es considerar a la dignidad humana, por un lado, como criterio para determinar principios, normas y valores y, por otro, como horizonte al que han de tender las acciones del ser humano (Yurén, 2008). Si la dignidad humana es criterio y horizonte, entonces la pregunta fundamental es cómo llegar a ella desde el campo educativo. La dignidad humana es un ideal del pensamiento crítico al cual nos acercamos mediante un proceso continuo de formación que implica elevar paulatinamente los niveles de conciencia, identidad, autocreación, libertad, conocimiento y sociabilidad en los educandos, como miembros constructivos del género humano y personas forjadoras de una cultura de la cual se benefician y a la cual sirven. 
Partiendo de lo anterior, conviene ahora responder ¿cuáles son las funciones de la reflexión crítica que propician procesos de formación escolar y por consiguiente de crecimiento personal? Anotemos algunos puntos significativos.

\section{NUEVAS PEDAGOGÍAS PARA UNA ÉPOCA DE CAMBIOS}

La necesidad cada vez más creciente de comprender y mejorar los procesos de aprendizaje para proyectar a los niños y jóvenes hacia caminos inéditos de crecimiento humano ha suscitado, desde los años ochenta, un renovado interés en el campo educativo en torno al valor y la necesidad de enseñar a pensar. Los aportes han llegado desde diferentes perspectivas. La psicología evolutiva, en general, y las investigaciones de Piaget sobre cómo evoluciona el pensamiento humano desde un nivel preoperatorio hasta alcanzar las formas superiores del razonamiento formal, en particular, han despertado mayor interés por educar en la capacidad de atender, entender y juzgar antes que en la de memorizar y repetir. Los nuevos paradigmas centrados en el aprendizaje cognitivo, constructivo, por descubrimiento, experiencial o basado en problemas se fundamentan en las conquistas de la investigación sobre el desarrollo del pensamiento alcanzadas por el movimiento cognitivo en los años ochenta y noventa (Montero, 1997, p. 14). A todo esto se suman los datos que aportan las nuevas investigaciones y teorías sobre la inteligencia y el funcionamiento de los dos hemisferios y las distintas zonas del cerebro: Edward de Bono y el pensamiento lateral desarrollado en el programa CORT (Cognitive Researb Team); Howard Gardner y la teoría de las inteligencias múltiples (1998); Reuven Feuerstein con el programa de enriquecimiento instrumental que se ha empleado con éxito en los colegios de Israel; Daniel Goleman y la inteligencia emocional que se popularizó en 1995; José Antonio Marina y la teoría de la inteligencia creadora, donde muestra la deslumbrante flexibilidad de la inteligencia humana; el aprendizaje basado en problemas (ABP), que busca enfrentar al estudiante a situaciones reales o hipotéticas donde tiene que procesar información para tomar decisiones acertadas en medio de un amplio espectro de posibilidades. Estas, entre otras teorías, desafían a la pedagogía y obligan a los educadores a trabajar con una visión centrada en la peculiaridad de las personas y con mayor énfasis en el potencial de desarrollo del pensamiento del hombre y la mujer, que en muchas ocasiones se deja de lado por la preocupación excesiva en los contenidos académicos (Montero, 1997).

Por otra parte, en el ámbito mundial se ha constatado la necesidad de desarrollar tres clases de conocimientos claves para el progreso efectivo de la humanidad, a saber: a) aquel que se requiere para la mejora continua de procesos, productos y servicios; b) el tipo de conocimiento que se utiliza para la explotación continua de conocimientos ya existentes, con el fin de mantener la producción de objetos y servicios que benefician la vida cotidiana; y c) el conocimiento que se emplea para producir innovación genuina y avance tecnológico (Rojas, 2006, p. 23). Esta constatación ha empujado a los educadores a estudiar cómo capacitar para que las alumnas y alumnos tengan autonomía para descubrir, construir y producir aprendizajes y conocimientos constantemente a lo largo de toda su vida. Así, como producto de una época de grandes cambios, lentamente se han impuesto las pedagogías enfocadas a "aprender a aprender", las cuales requieren de una formación especial en capacidades, habilidades y destrezas que deben conducir al dominio del pensamiento.

No obstante, cabe anotar que este proceso de aprender a aprender, tan unido a la cognición, no está desligado de la formación en valores: la vivencia de valores debe estar siempre sometida al tamiz del pensamiento crítico, así como el ejercicio de la crítica esta mediado por el horizonte de valores (Freire, 2004, p. 42). La unión de estas dos dimensiones, crítica y valorativa, se concretiza en los juicios que realizamos sobre la realidad superando el sentido común, la mera opinión e incluso la subjetividad que nos acompaña en todo momento. Al descubrir y afirmar algo como verdadero, la persona se responsabiliza de esta verdad y tiene que tomar una postura ante ella, lo que necesariamente lo llevará a tomar decisiones. Siendo así, el pensamiento crítico no es solo una forma de conocer el mundo, sino un camino de humanización. Estos dos elementos aquí mencionados, el pensamiento crítico como potencialidad y tendencia a conocer la realidad con verdad y el pensamiento crítico como factor de crecimiento humano, son los dos pilares que sostienen el punto de vista inicial. 


\section{PENSAMIENTO CRÍTICO Y ENSEÑANZA DE LA REFLEXIÓN}

Las definiciones que encontramos en textos e internet acerca del pensamiento crítico son numerosas y variadas. En lo que aquí concierne, solo asumiremos unas pocas nociones que son suficientes para desarrollar las ideas centrales que nos orientan. En 1999 el Dr. Huitt define el pensamiento crítico como "la actividad mental disciplinada de evaluar los argumentos o proposiciones haciendo juicios que puedan guiar el desarrollo de las creencias y la toma de acción” (p. 231); "La habilidad de analizar hechos, generar y organizar ideas, defender sus opiniones, hacer comparaciones, hacer inferencias, evaluar argumentos y resolver problemas" (Chance, 1995); "Es el pensamiento ordenado y claro que lleva al conocimiento de la realidad, por medio de la afirmación de juicios de verdad" (López Calva, 2003). Por su parte, Mathew Lippman, en su programa de filosofía para niños, propone que se trata de:

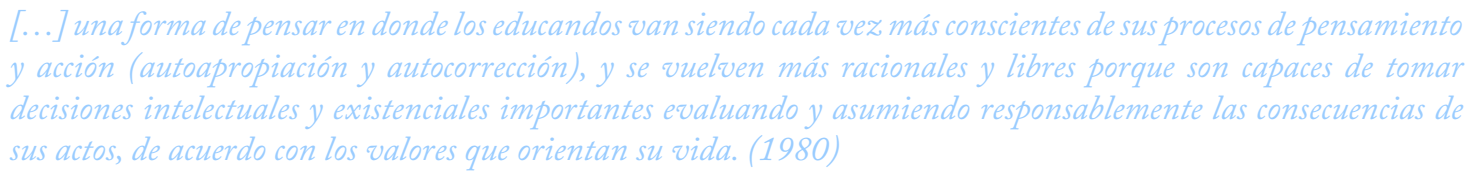

Estas son algunas definiciones que podemos encontrar sobre pensamiento crítico desarrolladas en las dos últimas décadas. En todas encontramos un elemento común: la capacidad para evaluar y cuestionar con criterios racionalmente justificados la validez de cualquier teoría, creencia, afirmación o fuente de información. En esta noción se recoge una importante transformación educativa para el siglo XXI: cambiamos de la enseñanza memorística al modelo de desarrollo de competencias, que cada día se abre paso en las escuelas de todo el mundo (Saiz, 2002, p. 57).

Si revisamos un poco el pasado, podemos afirmar que el pensamiento crítico como actividad mental no es nuevo; ya Sócrates tenía elementos de pensamiento crítico y, prácticamente, en toda la historia de la filosofía encontramos huellas de pensadores críticos. Ignacio Ellacuría, queriendo formar a los jóvenes salvadoreños en el pensamiento crítico en los años setenta, planteaba lo siguiente:

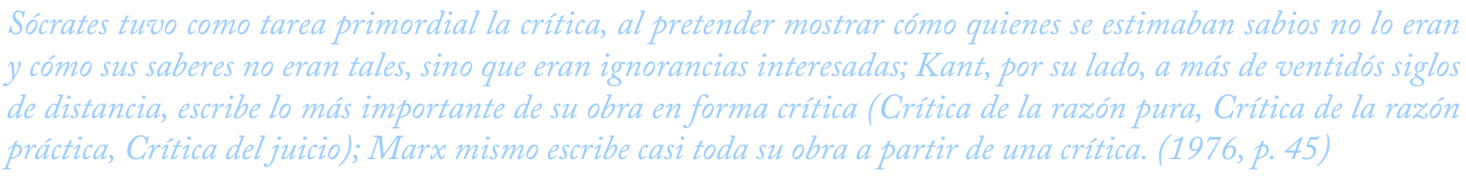

En realidad, casi todos los filósofos se debaten en permanente crítica unos de otros, lo cual viene sucediendo desde hace más de dos mil años. Lo que sí es nuevo es la gran relevancia que tiene hoy el pensamiento crítico dentro de los procesos educativos, lo cual indica que ha dejado de ser patrimonio de grandes intelectuales, para convertirse en una actitud cotidiana frente al conocimiento que puede ser adquirida desde temprana edad con una adecuada orientación en la escuela (Tuñón y Pérez, 2009, p. 156).

Con estos presupuestos, en adelante no conviene detenernos en un estudio psicológico o filosófico del tema, el cual es ampliamente tratado en textos y artículos especializados al alcance de todos; más bien profundizaremos sobre la

Panorama pp. $59-72 \mid$ Volumen 7 Número 13 | tesis propuesta inicialmente, donde se afirma la compleja y necesaria unión entre reflexión crítica y procesos de humanización.

Según Martín López Calva (2003), lo propio de la reflexión consiste en despertar ciertos dinamismos humanos mentales que todos poseemos, pero que no desarrollamos porque nadie nos orienta en la dirección adecuada para lograrlo. Esto no es extraño, pues como hemos visto en muchas ocasiones el talento y la genialidad se pierden cuando no se estimula al estudiante para que exteriorice todo aquello de lo que es capaz. De igual forma, el pensamiento crítico es una reflexión conducida intencionalmente para suscitar un tipo de ideas, juicios y visiones de la realidad que se caracterizan por poseer criterios racionalmente justificados. La escuela, por tanto, se convierte en un lugar apropiado para desarrollar todos aquellos dinamismos que posibilitan el surgimiento del pensamiento crítico. 
Entonces, ¿a qué nos referimos cuando decimos que hay que enseñar a reflexionar? No nos referimos al simple pensar, porque el simple pensar no necesita de la pedagogía, se hace inevitable y espontáneamente: la vida cotidiana tiene pensamiento y sentido común. Cuando hablamos de enseñar a reflexionar nos referimos a enseñar un pensar selectivo, a no ser dominados por el pensamiento, sino conducirlo para hacerlo propio o para dirigirlo hacia donde nosotros consideramos que es pertinente, significativo y productivo (Montero, 1997).

Si escudriñamos un poco descubrimos que el pensamiento crítico no es una tendencia alejada de lo que hoy en día llamamos competencias cognitivas, pues, en últimas, un pensador crítico debe manejar necesariamente habilidades tales como la interpretación, el analisis, la evaluación y la explicación, a la hora de producir buenos razonamientos que puedan afectar el mundo en que vivimos. Esto nos indica que el pensamiento crítico, si bien está latente, no es espontáneo o casual, sino producto juicioso de procesos educativos cuidadosamente planeados.

\section{FUNCIÓN CRÍTICA DE LA RELEXIÓN Y PROCESOS DE HUMANIZACIÓN}

El pensamiento humano tiene muchos condicionamientos, que van desde lo psicobiológico, pasando por las características de la cultura, hasta las condiciones materiales en las que los individuos están inmersos. No obstante, estos condicionamientos propios de la finitud humana, lejos de ser un obstáculo para la reflexión, se convierten en auténticos detonantes de procesos de liberación y racionalización del pensar humano en el pensamiento crítico. Las condiciones políticas, sociales, económicas y culturales, entre otras, constituyen ese complejo entramado que llamamos realidad circundante, que es precisamente el objeto de estudio del pensamiento crítico. La realidad es el insumo del quehacer crítico, al posibilitar que surja la duda, la negación, la eterna pregunta por el por qué, y al permitir, por consiguiente, que estalle la autonomía del pensamiento que no se resigna a lo dado, sino que busca alternativas, evalúa posiciones, pone en tela de juicio las teorías, explora nuevas ideas, construye mundos posibles, transforma la misma realidad.

Según esto, el pensamiento crítico no es la transmisión y el aprendizaje de contenidos más o menos entendidos; no es una nueva asignatura, sino la puesta en marcha de la autonomía del pensamiento, sobre todo en el momento actual donde el conocimiento deja de ser lento, escaso y estable y se percibe más bien como una realidad que está en continua expansión y renovación. Este hecho, como bien dice José Tejada Fernández, afecta a la educación, pues en la medida que la educación opera con el conocimiento, esta ha de plantearse siempre qué contenidos seleccionar, con qué criterios (actualidad, valía, relevancia, etcétera), y qué sistemas activar para su renovación o la integración de los nuevos saberes en la labor diaria de las instituciones, de tal manera que se superen los planteamientos anacrónicos en los que es fácil recaer (2000). Definitivamente, la educación se está transformando, y algunas de las respuestas que podemos brindar a las nuevas exigencias del contexto mundial las encontramos en los principios de actuación que resultan del análisis del pensamiento crítico como herramienta privilegiada en los procesos de enseñanza y aprendizaje del momento actual.

A continuación se presentan, a modo de promesas y de realidades en construcción, algunos de estos planteamientos: no vivimos en el sueño ilustrado de una sociedad auténticamente libre y reconciliada, o en el sapere aude proclamado por Kant (ten el valor de usar tu propia razón); incluso, muchos estudios bien fundamentados muestran que más del 60\% de los niños y jóvenes no utilizan el pensamiento crítico ni en la escuela ni en su vida diaria (Muñoz y Beltrán, 2001); lo cual indica que solo estamos al filo de la oportunidad de una renovación en nuestras prácticas pedagógicas, la cual esperamos que contribuya constructivamente al crecimiento humano en todos los niveles. 
Mediante la reflexión crítica podemos transformar la experiencia subjetiva en juicio e investigación razonada

Normalmente juzgamos las cosas según como nos ha ido con ellas, según la experiencia que tenemos de ellas. Sin embargo, la experiencia por sí misma no es suficiente para garantizarnos la conquista de la verdad, porque sabemos que toda experiencia es limitada, parcial, temporal, casi siempre vista desde una sola perspectiva (la del sujeto cognoscente), lo cual fácilmente nos puede conducir al subjetivismo epistemológico. "Es la reflexión la que posibilita el estudio, el análisis, la evaluación y el sentido consistente de la experiencia y da los elementos necesarios para el juicio” (Montero, 1997, p. 12). Los juicios son momentos significativos en nuestro pensar, son elementos del proceso de intelección para ascender por medio de la reflexión a otros pensamientos más complejos y profundos que nos llevan a las puertas de las decisiones y la acción. ${ }^{2}$ Con los juicios podemos superar los propios sentimientos e intereses, podemos comprendernos unos a otros, comprendernos como humanidad en proceso y como seres abiertos a la trascendencia (Lonergan, 1999).

Esto adquiere relevancia incluso en las elecciones cotidianas. En ocasiones, por la falta de reflexión nos dejamos guiar por las ciegas experiencias y las emotividades, y damos lugar a juicios equivocados que generan confusiones e interpretaciones erróneas de los hechos. La realidad política, económica y social de la nación, así como los acontecimientos mundiales, no pueden ser juzgados únicamente a la luz de las últimas noticias sensacionalistas, sino mediante rigurosos procesos de investigación razonada que nos amplíen la mirada, a veces unidireccional y efímera, del momento histórico que vivimos. Por experiencia sabemos que los estudiantes se guían por opiniones, sesgos y prejuicios que a veces escuchan de sus compañeros o que se fundamentan en datos adquiridos en la calle, pero el aprendizaje importante consiste, como decía Kant en los albores de la ilustración, en aprender a pensar por sí mismo con criterios racionalmente justificados y en situaciones específicas, con criterios éticamente sostenibles capaces de generar cambios significativos en la manera de pensar y proceder. Esta última anotación no es superflua, el pensamiento crítico es necesario para defender los valores humanos y la dignificación de la persona en momentos en que se levantan nuevos totalitarismos políticos que exigen obediencia ciega a reglas arbitrarias, fanatismos religiosos, exclusiones sociales y acciones concretas de devastación ecológica, donde importa poco que se pierda la armonía entre el hombre y la tierra, así como tampoco parece importar que con el tiempo no haya futuro para nadie (Freire, 2006).

\section{El pensamiento crítico posibilita el diálogo y la comprensión del otro}

La diferencia entre la conversación cotidiana y el verdadero diálogo racional radica en que este es un pensamiento estructurado, no centrado en los sentimientos y pasiones, orientado y focalizado en un problema frente al cual todos los participantes pueden interactuar en igualdad de condiciones exponiendo sus ideas y argumentos. Un educando que va siendo más crítico es un sujeto capaz de encontrar sentido o significado a las cosas en compañía, a partir de o junto con el otro (López Calva, 2003).

En la vida cotidiana vemos cómo los diálogos de nuestros estudiantes se caracterizan por la trivialidad, el vagabundeo de ideas y hasta el sinsentido, lo que llamamos charla de opinión o simple emisión de palabras y sentimientos. Dialogar en general y, sobre todo, dialogar intelectualmente en el aula es un aprendizaje que requiere de escucha y respeto,

Panorama pp. $59-72$ | Volumen 7 | Número 13 | también de cierto entrenamiento continuo para comprender aquello de lo que se está hablando con una actitud crítica capaz de evaluar y repasar aquello que se cree entender hasta lograr juicios concluyentes en torno a la discusión (Tuñón y Pérez, 2009, p. 157). De esta manera el pensamiento crítico forma en la actitud de participar democráticamente en la búsqueda de mejores opciones de vida justa y digna para todos, no importa que las ideas no sean siempre las propias. Si el estudiante ve críticamente mejores argumentos podrá comprenderlos y hacerlos suyos porque reconoce en ellos una racionalidad que supera los intereses y prejuicios particulares que naturalmente están presentes en toda discusión académica (Moguel, 2003).

2 Según el filósofo Bernard Lonergan (1999) el conocimiento es un proceso que se da a modo de escalera: en los peldaños inferiores encontramos la experiencia y el entendimiento, en los escalones superiores encontramos el juicio y la acción que serían propiamente los niveles a los cuales busca acceder el pensamiento crítico. 
De otra parte, en la educación superior el diálogo deja de ser un asunto netamente interpersonal para convertirse en diálogo intelectual, con una comunidad científica internacional que está abierta a la escucha pero también a la crítica y el debate sólidamente fundamentado. Por último, no debemos olvidar que el conocimiento compartido es una característica actual de las filosofías de gestión participativa. No obstante, cuando se carece de formación académica y de juicio crítico los individuos en las empresas solo pueden darle a sus conocimientos la frágil fundamentación de que estos han sido adquiridos en la dura escuela de la vida, respaldo que tiene validez solo en un entorno inmediato para resolver asuntos cotidianos, lo que no sucede cuando se trata de asuntos especializados donde el diálogo se centra en un reconocimiento de saberes y de juicios significativos adecuados para resolver los grandes problemas del sector productivo (Rojas, 2006).

\section{El pensamiento crítico es fundamental para dar el paso decisivo de una sociedad de la información a una sociedad del conocimiento}

A mediados de los años ochenta se fue imponiendo el término sociedad de la información para dar a entender toda la amplia gama de tecnologías de información y comunicación que vertiginosamente se abrían paso en el planeta transformando las costumbres y modos de vida. Hoy percibimos esta realidad en casi todos los ámbitos de la vida: transportes, compras, relaciones sociales, etcétera. La sociedad de la información es un hecho. No obstante, desde finales de los años noventa un nuevo término, sociedad del conocimiento, se impone como tendencia académica, económica y política en el ámbito mundial. Este término heurístico nos dice que no basta con la información que está al alcance de la mayoría con las TIC, sino que el nuevo capital intelectual radica en la capacidad para transformar esa información, a veces saturada e inconexa, en conocimiento útil, provechoso: "En el tercer milenio las actividades principales dedicadas a la creación de riqueza no serán la asignación del capital a la transformación de materia prima en productos elaborados, ni el trabajo mismo, sino la creatividad y la innovación, esto es, aplicaciones del conocimiento al trabajo" (Drucker, 1998). La información es mercancía valiosa, pero el conocimiento gobierna y decide sobre lo que se va a hacer con esta mercancía.

Es en este entorno actual, altamente especializado, donde el pensamiento crítico toma relevancia, pues la amplia gama de información recogida debe ser contrastada y analizada para determinar si el contenido de la información se ajusta o no a los hechos; si la información es verdadera, parcial, distorsionada, contradictoria o falsa y, sobre todo, qué posibilidades tenemos de transformar esa información recibida en ideas y proyectos nuevos, lo que denominamos innovación y gestión del conocimiento (Páez, Arreaza y Vizcaya, 2005, pp. 239-241).

\section{La reflexión que sustenta el pensamiento crítico permite determinar las líneas concretas de acción para transformar la realidad}

Todo proceso de reflexión crítica debe concluir en una previsión para el futuro, mostrando las diferentes alternativas posibles y definiendo líneas concretas de acción que permitirán variar una situación determinada. Esto significa que el pensamiento crítico nos garantiza desechar definitivamente el dogmatismo que se guía por esquemas rígidos, sin tener en cuenta la dinámica social que cambia permanentemente. Siendo así, cualquier visión acrítica de la realidad tenderá inevitablemente a convertirse en una reafirmación de principios generales que fácilmente se convierten en consignas y eslóganes prefabricados que se imponen. Esto es lo que encontramos en las frases grandilocuentes de los gurús de la moda o del mundo de los negocios, los especialistas que rinden culto al cuerpo e indican cuál es el patrón a seguir para tener una figura aceptable, las ideas de los publicistas que nos dicen aquello que debemos consumir, así como también las viejas y nuevas manipulaciones de los políticos que encuentran la manera de convencer a un pueblo pasivo e indiferente frente a lo que pasa (Elder y Paul, 2003, pp. 16-18).

Según lo anterior, el pensamiento crítico, y los correspondientes procesos de reflexión que este implica, no es una tarea reservada e importante solo para los cuadros dirigentes de las organizaciones, o algo tan difícil de desarrollar que únicamente algunos especialistas lo pueden realizar. Al contrario, lo que aquí se plantea es que el pensamiento crítico 
hoy es una necesidad vital en los procesos educativos, si de verdad queremos formar jóvenes para una participación consciente y decidida en los urgentes cambios sociales, económicos, científicos y culturales que el país necesita (Prieto, 2008, pp. 4-5).

Iván Alfonso

Pinedo

Esta última idea indica que el pensamiento crítico no es un patrimonio exclusivo del mundo académico, sino que correctamente orientado es capaz de afectar un amplio espectro de situaciones claves de la vida cotidiana y de la cultura: el pensamiento crítico puede empezar en el aula, pero va más allá de esta, pues el objetivo último consiste en que el estudiante pueda desarrollar en todos los aspectos de su vida la disposición hacia el pensamiento crítico. Estos planteamientos contrastan con la realidad de muchas escuelas, donde se percibe el profundo abismo entre el pensamiento producido por la repetición de información y el pensamiento requerido para tomar decisiones en el mundo real (Sierra, Carpintero y Pérez, 2010, p. 99).

Recientemente el filósofo Guillermo Hoyos, uno de los más notables pensadores colombianos de los últimos tiempos, comentaba en el diario El Tiempo como hacía falta en las escuelas una cátedra de cultura política y convivencia ciudadana que posibilitara el diálogo crítico en torno a lo que sucedía en el ámbito local y transnacional en el surcontinente. En democracias que aún están en construcción, donde muchas cosas parecen dar igual, donde existe la sensación de que todo es válido y todo se puede con tal de alcanzar los objetivos propuestos ¿no es necesario un pensamiento crítico urgente que cuestione cientos de prácticas políticas, económicas y sociales que han sido aceptadas acríticamente, al menos por dos generaciones que se han caracterizado porque no les interesaba el cambio o que quizá no sabían qué preguntas hacerse ni tampoco cómo sustituir el pensamiento vigente por uno más fiable? Nada distante de lo que Edgar Morin ha propuesto sobre la necesidad de una "refundación" de la educación, caracterizada por una crisis de sentido y una carencia cada vez mayor de actitud interrogativa que olvida el valor epistemológico de la duda en la construcción del conocimiento.

\section{La reflexión crítica es fundamental para darle un sentido global-existencial a la vida}

Saber dónde estamos, hacia dónde vamos, cuál es el mejor camino y cuál nos aleja de la meta, solo es posible con la fluorescencia de la reflexión. Desde una perspectiva existencialista, el ser humano no solo es lo que es ahora, sino lo que puede ser en el futuro. El colegio, por tanto, no es solo el lugar de los conocimientos académicos, sino el lugar para abrirse a valores universales, creencias y convicciones que dan el sentido global y profundo de la experiencia, de la propia vida, y desde ella, al mundo, la historia y la cultura. Una buena reflexión puede ser el detonante de procesos inéditos de superación y de un movimiento hacia fuera de sí mismo que puede llevar a cualquier persona a decir: "yo antes era así, pero ahora soy así y quiero en el futuro ser de tal manera". Sartre decía "el hombre no nace sino que se hace", indicando que no hay un determinismo, no hay un modelo de hombre establecido de antemano, sino que cada cual debe hacer su proyecto de hombre, pero si la escuela no contribuye al logro de esas ilusiones personales, aspiraciones existenciales o potencialidades mentales, entonces cabe hacerse la pregunta: ¿para qué educamos? (Padilla, 2007).

Son muchas, pues, las ideas que el pensamiento crítico suscita y deja como inquietud. En esta exposición el centro

Panorama pp. $59-72 \mid$ Volumen 7 | Número 13 fue la humanización, asunto que no es trivial ni descontextualizado de la realidad colombiana. Para finalizar, conviene comentar una breve idea surgida en el Foro Mundial sobre Educación en Dakar, en el año 2000, que tenía como motivación central "la educación para todos" y cuyas memorias pueden ser consultadas en internet. En este foro, y más tarde en otros estudios sobre equidad y políticas educativas (Mancebo y Goyeneche, 2010), se afirmaba en algunos países se daba un extraño fenómeno educativo denominado "exclusión social por inclusión en el sistema educativo", lo cual, en últimas, era una situación paradójica. Esta exclusión consistía en lo siguiente: en muchas naciones hay un afán por la cobertura escolar, lo cual es un esfuerzo pertinente, pero sucede que para muchos estudiantes, que pasan diez años en una escuela, al finalizar, el resultado de ese tránsito por el sistema educativo es una preparación para quedar excluidos dentro de la sociedad, pues en el ámbito académico el estudiante no aprendió lo que debía aprender o adquirió aprendizajes de baja relevancia (Gentile, 2009); en el moral no adquirió los valores esperados y en el emocional o espiritual no creció como persona, entonces salía de la escuela para ser un relegado más dentro del orden social vigente. 
Esto, por supuesto, es inaceptable desde todo punto de vista pedagógico. Un padre envía a sus hijos al colegio con la esperanza de un excelente proceso de humanización, tal como aquí se ha propuesto, pero si ese es el resultado, entonces ¿qué pasó en la escuela año tras año? Son experiencias que nos cuestionan y nos invitan a la reflexión sobre nuestro quehacer docente en una profesión y vocación que, no obstante las múltiples dificultades que a veces encontramos, es decisiva para construir una Colombia que realmente sea una casa del conocimiento, abierta e inteligente para todos.

El ideal

de pensar críticamente: promesas $y$ realidades
| Panorama

| pp. 59 - 72

| Volumen 7

| Número 13

| Julio - diciembre

| 2013 


\section{REFERENCIAS}

Iván Alfonso

Pinedo Cantillo |

2. Bono, E. de (1991). Aprender a pensar. Barcelona, España: Plaza y Janés.

3. Chance, P. (1995). Aprendizaje y conducta. México DF, México: Manual Moderno.

4. Derek, E. (1988). El conocimiento compartido. El desarrollo de la comprensión en el aula. Barcelona, España: Paidós.

5. Drucker, P. (1998). La sociedad postcapitalista. Bogotá, Colombia: Norma.

6. Elder, L. y Paul, R. (2003). La mini guía para el pensamiento crítico: conceptos y herramientas. California: Fundación para el pensamiento crítico. Recuperado de: http://www.criticalthinking.org/ resources/PDF/SP-ConceptsandTools.pdf

7. Ellacuría, I. (1976). ¿Filosofía, para qué? Abra, 11, 42-48.

8. Facione, P. (2007). Critical thinking: what it is and why it counts. Milbrae, CA: The California Academic.

9. Fernández Tejada,J. (2000). La educación en el marco de una sociedad global: algunos principios y nuevas exigencias. Profesorado. Revista de Curriculum y formación de profesorado, 4(1), 1-11.

10. Freire, P. (2004). Pedagogía de la autonomía. Sao Paulo, Brasil: Paz e Terra.

11. Froemel, J. (2006). Los estudios internacionales del rendimiento educativo y los países en vía de desarrollo: participación resultados y relevancia. Revista de Educación (número especial), 131-152.

12. Gardner, H. (1998). Inteligencias múltiples. Barcelona, España: Paidós.

13. Gentile, P. (2009). Marchas y contramarchas. El derecho a la educación y las dinámicas de exclusión incluyente en América Latina (a sesenta años de la Declaración Universal de los Derechos Humanos). Revista Iberoamericana de Educación, 49, 19-57.

14. Huitt, W. (1999). El pensamiento crítico. Recuperado de: http://teach.valdosta.edu/whuitt/edpsyppt/.../ critical\%20thinking\%20sp.pp.

15. Instituto Colombiano para el Fomento de la Educación Superior, Icfes (2010). Colombia en PISA 2009. Sintesis de resultados. Bogotá, Colombia: Cadena.

Panorama pp. $59-72$ Volumen 7 Número 13

18. Lippman, M. (1991). Thinking in education. Cambridge, UK: University Press.

19. Lippman, M. (1998). Pensamiento complejo y educación. Madrid, España: De la Torre.

16. Instituto Colombiano para el Fomento de la Educación Superior, Icfes (2010). Resultados de Colombia en TIMSS 2007. Resumen ejecutivo (2a ed.). Bogotá, Colombia: Cadena.

17. Lippman, M. (1980). Philosophy in the classroom. Filadelfia, PA: Themple University Press. 
22. Mancebo, E. y Goyeneche, G. (2010). Las políticas de inclusión educativa: entre la exclusión social y la innovación pedagógica. IX Jornadas de la Facultad de Ciencias Sociales de la Universidad de la República. Montevideo, Uruguay. Recuperado de: http://www.fcs.edu.uy/archivos/Mesa_12_y_17_ManceboGoyeneche.pdf

23. Martin, M. O.; Mullis, I. V.y Foy, P. (2008). TIMSS 2007 International science report. Boston, MA: TIMSS \& PIRLS International Study Center Lynch School of Education, Boston College.

24. Moguel, D. (2003). Effective classroom discussions: getting teachers to talk less and students to talk more. Social Studies Review, 42(2), 96.

25. Montero, J. (1997). La reflexión como objetivo actual de la pedagogía. Asunción, Paraguay: CPAL.

26. Montoya,J. (2007). Acercamiento al desarrollo del pensamiento crítico, un reto para la educación actual. Recuperado de: http://formacioncontinuaedomex.files.wordpress.com/2011/06/montoya-javier-investigacic3b3n_pensamiento_crc3adtico.pdf

27. Morin, E. (1999). Los siete saberes necesarios para la educación del futuro. Bogotá, Colombia: Ministerio de Educacion Nacional-Unesco.

28. Mullis, I. V.; Martín, M. O.; González, E. J., y Chrostowski, S. J. (2008). TIMSS 2007 International Mathematics Report. Boston, MA:TIMSS \& PIRLS International Study Center Lynch School of Education, Boston College.

29. Muñoz, A. y Beltran,J. (2001). Fomento del pensamiento crítico mediante la intervención en una unidad didáctica sobre la técnica de la detección de información sesgada en los alumnos de enseñanza secundaria obligatoria en ciencias sociales. CIOPA 2001 Congreso Internacional on line de Psicología Aplicada. Recuperado de: http://www.psicologia-online.com/ciopa2001/actividades/54/index.html

30. Organización para la Cooperación y el Desarrollo Económicos, OECD (2004). First result from PISA 2003. París, Francia: OECD.

31. Organización para la Cooperación y el Desarrollo Económicos, OECD; Programme for International Student Assessment, PISA (2010). PISA 2009 Results. Learning trends. Changes in student performance since 2000 (vol. V). París, Francia: OECD.

32. Organización para la Cooperación y el Desarrollo Económicos, OECD; Organización de las Naciones Unidas para la Educación, la Ciencia y la Cultura, Unesco (2003). Literacy skills for the world of tomorrow. Further results from PISA 2000. Paris: OECD-Unesco Institute for statistics.

33. Padilla, C. (coord.) (2007). Factores que influyen en el desarrollo del pensamiento crítico: representaciones estudiantiles, Primeras Jornadas de Lectura y Escritura. Lectura y escritura críticas: perspectivas múltiples. Cátedra Unesco. Tucumán, Argentina; Universidad Nacional de Tucumán. Recuperado de: http://www.filo.unt.edu.ar/jorn_unesco/cd/PAN\%203.1.DOUGLAS.pdf

34. Páez,H.; Arreaza, E. y Vizcaya,W. (2005). Educar para pensar críticamente: una visión desde el área curricular Estudios Sociales de Educación Básica. Revista de Teoría y Didáctica de las Ciencias Sociales, 10, 237-263.

35. Prieto, J. M. (2008). Pensamiento crítico y universidad: estrategias para la consolidación de una sociedad democrática en México. Investigación y Ciencia de la Universidad Autónoma de Aguascalientes, 42, 36-44. Acodesi. 
37. Rojas, J. (2006). Gestión educativa en la sociedad del conocimiento. Bogotá, Colombia: Magisterio.

38. Saiz, C. (2002). Enseñar o aprender a pensar. Escritos de Psicología, 6, 53-72.

Iván Alfonso

Pinedo

Cantillo | 40. Sierra, J.; Carpintero, E. y Pérez, L. (2010). Pensamiento crítico y capacidad intelectual. Faísca, 15(17),
98-110.

41. Tuñón, M. C. y Pérez, M. (2009). Características del discurso en el aula de clase como mediación para el desarrollo de pensamiento crítico. Zona Próxima, 11, 144-159.

42. Yurén, M. T. (2008). La filosofía de la educación en México. Principios, fines y valores. México DF, México: Trillas.

39. Saiz, C. y Nieto, A. (2002). Pensamiento crítico, capacidades y desarrollo: conceptos básicos y actividades prácticas. Madrid, España: Pirámide.
Panorama

pp. 59 - 72 |

Volumen 7 |

Número 13

Julio - diciembre |

2013 | 